\title{
On the space of elliptic genera
}

\author{
JAN MANSChOT
}

\begin{abstract}
Invariance under modular transformations and spectral flow restrict the possible spectra of superconformal field theories (SCFTs). This paper presents a technique to calculate the number of constraints on the polar spectra of $\mathcal{N}=(2,2)$ and $\mathcal{N}=(4,0)$ SCFTs by analyzing their elliptic genera. The polar spectrum corresponds to the principal part of a Laurent expansion derived from the elliptic genus. From the point of view of the $\mathrm{AdS}_{3} / \mathrm{CFT}_{2}$ correspondence, these are the states which lie below the cosmic censorship bound in classical gravity. The dimension of the space of elliptic genera is obtained as the number of coefficients of the principal part minus the number of constraints. As an additional illustration of the technique, the constraints on the spectrum of $\mathcal{N}=4$ topologically twisted Yang-Mills on $\mathbb{C P}^{2}$ are discussed.
\end{abstract}

1. Introduction

2. $\mathcal{N}=(2,2)$ elliptic genera and Jacobi forms

3. Cusp forms as constraints on $\mathcal{N}=(2,2)$ spectra

4. Constraints on $\mathcal{N}=(4,0)$ spectra

5. Conclusion

References

\section{Introduction}

Because two-dimensional conformal field theories (CFTs) are diffeomorphism invariant, partition functions of CFTs are modular invariant [1]. This invariance has major implications for the spectrum of the CFT. An example 
is the asymptotic growth of degeneracies which is given by the celebrated Cardy formula. Modular invariance also imposes constraints on the part of the spectrum with a small number of degeneracies (compared to the regime of validity of the Cardy formula). Examples of such constraints are charge sum rules in superconformal field theories (SCFT) [2], and constraints on topological quantities of the target manifold when a sigma model is considered [3-5]. In these cases, the constraints are derived by an analysis of the elliptic genus, which counts states with an alternating sign dependent on the fermion number. The elliptic genus, being a supersymmetric index, enumerates BPS states and is well-protected against continuous changes of parameters which preserve supersymmetry. This makes the elliptic genus an important tool for the microscopic account of, for example, the entropy of D1-D5 brane systems [6] and M-theory black holes [7].

The present paper continues the study of implications on SCFT spectra by an analysis of the $\mathcal{N}=(2,2)$ and $\mathcal{N}=(4,0)$ elliptic genus. The symmetries of the elliptic genus can be derived from modular invariance and the spectral flow symmetry of the SCFT. It is shown that these symmetries impose constraints on the polar spectrum, ${ }^{1}$ whose degeneracies are generically small. Section 2 explains the notion of "polar spectrum." In the context of the $\mathrm{AdS}_{3} / \mathrm{CFT}_{2}$ correspondence [8], the polar spectrum is that part of the spectrum which classically lies below the cosmic censorship bound of $\mathrm{AdS}_{3}$-gravity [9]. This part of the spectrum is of particular importance in [10], where a gravity interpretation is given to the partition function of the boundary SCFT using the method of images. Manschot and Moore [11] show that not every set of polar degeneracies can be completed to a full partition function by this method. Certain spectra are thus easily excluded as CFT spectra, based on their inconsistency with the required symmetries. Gaberdiel et al. apply [13] the presence of constraints on $\mathcal{N}=(2,2)$ polar spectra to analyze the consistency of "pure" $\mathcal{N}=2$ supergravity with the SCFT symmetries. Of course, the existence of a partition function with the right properties does not imply the existence of a CFT. In case a CFT does exist and constraints are present, only a subset of the polar degeneracies need to be specified to determine the complete partition function. For large central charges of $\mathcal{N}=(2,2)$ and $\mathcal{N}=(4,0)$ SCFTs, the ratio of the number of polar degeneracies and the number of constraints is shown to grow linearly with the central charge.

\footnotetext{
${ }^{1}$ When the words "degeneracy" or "spectrum" are used in the following, counting of the states with $(-1)^{F}$ is implicitly assumed.
} 
Consistency of a given polar spectrum with the symmetries can be tested by the construction of a function (a vector-valued cusp form ${ }^{2}$ ), which is determined by the polar degeneracies. When this function is non-vanishing, no partition function with the right properties exists. This is explained in [11], which relied on methods described in [12]. The number of constraints on the polar spectrum is equal to the dimension of the space of appropriate vector-valued cusp forms. The present paper describes a technique, following the original work of Skoruppa [15], to calculate the dimension of the space of such cusp forms. By a more straightforward approach, simpler expressions for the dimension formulas for vector-valued cusp forms are derived compared to the formulas presented in [15].

As explained in Section 2, an $\mathcal{N}=(2,2)$ elliptic genus is an element of the space of weak Jacobi forms $\tilde{J}_{0, m}$ with an integral Fourier expansion. We will often refer to the space $\tilde{J}_{0, m}$ as the space of elliptic genera, although not even all functions with an integral expansion in $\tilde{J}_{0, m}$ appear as SCFT elliptic genera. Having obtained the dimension of the space of vector-valued cusp forms, one can calculate the dimension of the space of elliptic genera straightforwardly. The final expression for $\operatorname{dim} \tilde{J}_{0, m}$ (Equation (3.22)) equals the dimension formula given in [14]. However, the derivation here is qualitatively different. The technique described here has the advantage that it is more generally valid than the technique of [14], in physical applications where modular forms appear. Section 4 applies the technique to elliptic genera of $\mathcal{N}=(4,0)$ SCFTs, which are relevant for the microscopic explanation of the entropy of M-theory black holes [7,17-19]. As an additional illustration, the dimension of the space of weakly holomorphic functions is calculated, which satisfy the expected transformation properties of the partition functions of topologically twisted $\mathcal{N}=4$ supersymmetric $\operatorname{SU}(N)$ Yang-Mills. The constraints are more restrictive in this case than for the $\mathcal{N}=(4,0)$ SCFTs arising in M-theory. Another application is the calculation of the dimension of spaces of characters in rational CFTs, which is not attempted here.

Section 2 starts with a review of the $\mathcal{N}=(2,2)$ elliptic genus and its connection with weak Jacobi forms. The number of independent constraints is shown to equal the dimension of a certain space of vector-valued cusp forms in Section 3. The dimension of the space of cusp forms is calculated. The number of polar degeneracies minus this dimension gives the dimension

\footnotetext{
${ }^{2}$ Cusp forms are holomorphic modular forms which vanish at the cusps, which are the points $i \infty \cup \mathbb{Q}$.
} 
of the space of elliptic genera. The application to $\mathcal{N}=(4,0)$ elliptic genera and $\mathcal{N}=4$ Yang-Mills theory is discussed in Section 4.

\section{2. $\mathcal{N}=(2,2)$ elliptic genera and Jacobi forms}

The elliptic genus is defined as the trace over the Ramond-Ramond sector of an SCFT,

$$
Z(\tau, z)=\operatorname{Tr}_{\mathrm{RR}}(-)^{F} y^{J_{0}} q^{L_{0}-c_{L} / 24} \bar{q}^{\bar{L}_{0}-c_{R} / 24}
$$

where $q=e(\tau),{ }^{3} \tau \in \mathcal{H}$, where $\mathcal{H}$ is the upper-half plane, and $y=e(z), z \in$ $\mathbb{C}$. The insertion of $(-)^{F}$ (with $F=\frac{1}{2}\left(J_{0}-\bar{J}_{0}\right)$ being the fermion number) projects the trace on states which preserve the supersymmetries in the rightmoving sector. Since these are the right-moving ground states, the trace is independent of $\bar{\tau}$.

Modular invariance of the CFT implies that $Z(\tau, z)$ transforms under a modular transformation as [20]

$$
Z\left(\gamma(\tau), \frac{z}{j(\gamma, \tau)}\right)=e\left(\frac{m c z^{2}}{j(\gamma, \tau)}\right) Z(\tau, z), \quad \gamma=\left(\begin{array}{ll}
a & b \\
c & d
\end{array}\right) \in \Gamma, \quad m=\frac{c_{L}}{6}
$$

where $j(\gamma, \tau)=c \tau+d$, and $\Gamma$ is an abbreviation for $S L(2, \mathbb{Z})$. When the SCFT is a sigma model with $d$-dimensional target space, then $m=\frac{d}{4}$. Restricting to specific values of $z$ in this case gives several topological quantities of the target manifold [4].

Spectral flow is a symmetry of the superconformal algebra, which relates states with different periodicities of the fermions. States in the Ramond sector can for example be mapped to states in the Neveu-Schwarz sector. The requirement that the spectrum satisfies this symmetry implies that $Z(\tau, z)$ is quasi-periodic as a function of $z[20]$. The quasi-periodicity is given by

$$
Z(\tau, z+\lambda \tau+\mu)=(-1)^{2 m(\lambda+\mu)} e\left(-m\left(\lambda^{2} \tau+2 \lambda z\right)\right) Z(\tau, z), \quad(\lambda, \mu) \in \mathbb{Z}^{2}
$$

This paper considers elliptic genera with integer $m$; the results are easily generalized to the case of non-integer $m$. The symmetries (2.2) and (2.3) determine that the elliptic genus is a (weak) Jacobi form of weight 0 and index $m$ [14]. The adjective "weak" will be explained below. The space of weak Jacobi forms of weight 0 and index $m$ is denoted by $\tilde{J}_{0, m}$.

\footnotetext{
${ }^{3}$ We use the convention $e(x)=e^{2 \pi i x}$.
} 
Many aspects of Jacobi forms can be understood via the connection between Jacobi forms and vector-valued modular forms of half-integer weight. This connection is briefly outlined here; additional details and proofs can be found in [14]. A weak Jacobi form $\phi(\tau, z)$ has a Fourier expansion in terms of the integer coefficients $c(n, l)$

$$
\phi(\tau, z)=\sum_{n \geq 0, l \in \mathbb{Z}} c(n, l) q^{n} y^{l} .
$$

Spectral flow (2.3) determines $c(n, l)$ to be a function of only $4 m n-l^{2}$ and the residue $l \bmod 2 m$. The adjective "weak" in "weak Jacobi form" is used when $c(n, l)$ is non-zero for $4 m n-l^{2} \geq-m^{2}$, as opposed to $\geq 0$ for a Jacobi form. From spectral flow symmetry, or equivalently quasi-periodicity, one can deduce that $\phi(\tau, z)$ admits a decomposition into a set of functions $h_{\mu}(\tau)$ and theta functions $\theta_{m, \mu}(\tau, z)$ with $\mu \in \mathbb{Z} / 2 m \mathbb{Z}$. In terms of these functions, $\phi(\tau, z)$ is given by

$$
\phi(\tau, z)=\sum_{\mu} h_{\mu}(\tau) \theta_{m, \mu}(\tau, z) .
$$

The functions $h_{\mu}(\tau)$ and $\theta_{m, \mu}(\tau, z)$ are given explicitly by

$$
h_{\mu}(\tau)=\sum_{n=-\mu^{2}} c_{\mu}(n) q^{n / 4 m}, \quad \theta_{m, \mu}(\tau, z)=\sum_{\substack{l \in \mathbb{Z} \\ l=\mu \\ \bmod 2 m}} q^{l^{2} / 4 m} y^{l}
$$

where $c_{\mu}(n)=(-1)^{2 m l} c\left(\frac{n+l^{2}}{4 m}, l\right)$, and $l=\mu \bmod 2 m$. The domain of $\mu$ in Equation $(2.5)$ is taken to be $[-m+1, m]$.

All the information concerning the Fourier coefficients of $\phi(\tau, z)$ is thus captured in $h_{\mu}(\tau)$. The space $\widetilde{J}_{0, m}$ is therefore isomorphic to the space of appropriate vector-valued modular forms. The precise transformation properties of the vector $h_{\mu}(\tau)$ are described later in this section. The adjective "weak" implies that the Laurent expansion of $h_{\mu}(\tau)$ may admit a principal part, which corresponds to the terms with negative exponents, $-m / 4 \leq n / 4 m<0$. The negative exponents lead to a pole of $h_{\mu}(\tau)$ in the limit $\tau \rightarrow i \infty$. By $\Gamma$-transformations, the limit $\tau \rightarrow i \infty$ of the vector $h_{\mu}(\tau)$ is equivalent to $\tau \rightarrow \mathbb{Q}$. Modular forms which are only meromorphic for $\tau \rightarrow i \infty \cup \mathbb{Q}$ are called "weakly holomorphic". The poles of the $h_{\mu}(\tau)$ are weak enough to be canceled by the theta functions, such that $\phi(\tau, z)$ is an analytic function. The coefficients $c_{\mu}(n), n<0$, are referred to as "polar coefficients." We denote the space of polar coefficients for a given index $m$ 
by $P(m)$; the dimension of $P(m)$ is $p(m)$. These coefficients correspond to the terms with $4 m n-l^{2}<0$ in expansion (2.4).

The polar spectrum is defined as the set of states which is counted by the principal part of $h_{\mu}(\tau)$. From the point of view of the SCFT, the condition $4 m n-l^{2}<0$ corresponds to states, with eigenvalues $\frac{2}{3} c_{L}\left(L_{0}-\frac{c_{L}}{24}\right)-J_{0}^{2}<$ 0 . The notion of "polar spectrum" is more generally valid in CFT, for example the polar spectrum of a bosonic CFT is the set of states with $L_{0}-\frac{c_{L}}{24}<0$. In $\mathrm{AdS}_{3}$, a black hole with such quantum numbers would classically lead to a naked singularity. Therefore, the polar states lie below the cosmic censorship bound from the viewpoint of $\operatorname{AdS}_{3}$ gravity $[9,10]$. These states are interpreted as excitations of thermal $\mathrm{AdS}_{3}$, whereas the non-polar states are mostly contributed to black hole geometries [10,23].

The functions $h_{\mu}(\tau)$ and $\theta_{m, \mu}(\tau, z)$ transform under $\Gamma$ with half-integral weight, which requires the appearance of non-trivial unitary factors in modular transformations [21]. Therefore, a double sheeted cover of $\Gamma$, the metaplectic group $\tilde{\Gamma}$, is first introduced. An element $\tilde{\gamma} \in \tilde{\Gamma}$ is represented by

$$
\tilde{\gamma}=(\gamma, \epsilon \sqrt{j(\gamma, \tau)}), \quad \gamma \in \Gamma, \epsilon= \pm 1
$$

The square root of $j(\gamma, \tau)$ is defined unambiguously by requiring that the argument of a complex number $z$ lies in the domain $(-\pi, \pi]$. The product of two elements is defined by

$$
(\gamma, \epsilon \sqrt{j(\gamma, \tau)}) \cdot\left(\gamma^{\prime}, \epsilon^{\prime} \sqrt{j\left(\gamma^{\prime}, \tau\right)}\right)=\left(\gamma \gamma^{\prime}, \epsilon \epsilon^{\prime} \sqrt{j\left(\gamma, \gamma^{\prime}(\tau)\right)} \sqrt{j\left(\gamma^{\prime}, \tau\right)}\right)
$$

We define the slash operator $\left.\right|_{w} \tilde{\gamma}$ on a modular form $f(\tau)$ of (possibly halfinteger) weight $w$, by

$$
\left.f\right|_{w} \tilde{\gamma}=\epsilon^{-2 w} j(\gamma, \tau)^{-w} f(\gamma(\tau))
$$

and the slash operator $\left.\right|_{k, m} \tilde{\gamma}$ on Jacobi forms $\phi(\tau, z)$ of weight $k$ and index $m$ by

$$
\left.\phi\right|_{k, m} \tilde{\gamma}=\epsilon^{-2 k} j(\gamma, \tau)^{-k} e\left(-\frac{m c z^{2}}{j(\gamma, \tau)}\right) \phi\left(\gamma(\tau), \frac{z}{j(\gamma, \tau)}\right) .
$$


The set of theta functions $\theta_{m, \mu}$ transforms as a vector-valued Jacobi form under transformations $\tilde{\gamma} \in \tilde{\Gamma}$ :

$$
\left(\begin{array}{c}
\left.\theta_{m,-m+1}\right|_{\frac{1}{2}, m} \tilde{\gamma} \\
\left.\theta_{m,-m+2}\right|_{\frac{1}{2}, m} \tilde{\gamma} \\
\ldots \\
\cdots \\
\left.\theta_{m, m}\right|_{\frac{1}{2}, m} \tilde{\gamma}
\end{array}\right)=\mathbf{M}_{2 m}^{\mathrm{T}}(\tilde{\gamma})\left(\begin{array}{c}
\theta_{m,-m+1} \\
\theta_{m,-m+2} \\
\ldots \\
\ldots \\
\theta_{m, m}
\end{array}\right)
$$

where $\mathbf{M}_{2 m}(\tilde{\gamma})$ is a $2 m \times 2 m$ matrix. The matrix $\mathbf{M}_{2 m}(\tilde{\gamma})$ appears transposed in (2.11) for notational clarity in the rest of the text.

Generators of $\tilde{\Gamma}$ are $S=\left(\left(\begin{array}{cc}0 & -1 \\ 1 & 0\end{array}\right), \sqrt{\tau}\right)$ and $T=\left(\left(\begin{array}{ll}1 & 1 \\ 0 & 1\end{array}\right), 1\right)$. The transformations of $\theta_{m, \mu}(\tau, z)$ under $S$ and $T$ are given by

$$
\begin{aligned}
\left.\theta_{m, \mu}\right|_{1 / 2, m} S & =\frac{1}{\sqrt{2 m i}} \sum_{\nu} e\left(-\frac{\mu \nu}{2 m}\right) \theta_{m, \nu}(\tau, z), \\
\left.\theta_{m, \mu}\right|_{1 / 2, m} T & =e\left(\frac{\mu^{2}}{4 m}\right) \theta_{m, \mu}(\tau, z) .
\end{aligned}
$$

These transformations are in principle sufficient to deduce $\mathbf{M}_{2 m}(\tilde{\gamma})$ for general $\tilde{\gamma} \in \tilde{\Gamma}$. Closed expressions are also known [15]. For elements $\tilde{\gamma} \in \tilde{\Gamma}$ which lie in the congruence subgroup $\Gamma(4 m)^{*} \in \tilde{\Gamma}, \mathbf{M}_{2 m}(\tilde{\gamma})$ is the identity matrix. The group $\Gamma(4 m)^{*}$ is defined by

$$
\Gamma(4 m)^{*}=\left\{\tilde{\gamma}=\left(\gamma,\left(\frac{c}{d}\right) \varepsilon_{d}^{-1} j(\gamma, \tau)^{1 / 2}\right) \mid \gamma \in \Gamma(4 m)\right\}
$$

where $\Gamma(N) \in \Gamma$ is the principal congruence subgroup

$$
\Gamma(N)=\left\{\left(\begin{array}{ll}
a & b \\
c & d
\end{array}\right) \in \Gamma,\left(\begin{array}{ll}
a & b \\
c & d
\end{array}\right)=\left(\begin{array}{ll}
1 & 0 \\
0 & 1
\end{array}\right) \quad \bmod N\right\} .
$$

The groups $\Gamma(4 m)^{*}$ and $\Gamma(N)$ are normal subgroups of respectively $\tilde{\Gamma}$ and $\Gamma$. In $(2.13),\left(\frac{c}{d}\right)$ is the extended Legendre symbol $[21]$ and $\varepsilon_{d}=\sqrt{\left(\frac{-1}{d}\right)}$,

$$
\varepsilon_{d}=\left\{\begin{array}{lll}
1, & d=1 & \bmod 4 \\
i, & d=3 & \bmod 4
\end{array}\right.
$$

Equation (2.13) gives an explicit expression for $\epsilon$ in Equation (2.7). This expression is derived from the transformation properties of the theta function $\Theta(\tau)=\sum_{n \in \mathbb{Z}} q^{n^{2}}$ under $\Gamma_{0}(4)$, and is therefore consistent with the 
transformations of half-integer weight forms [21]. Since this expression for $\epsilon$ takes values in $( \pm 1, \pm i), \Theta(\tau)$ actually transforms under a four-sheeted cover of $\Gamma$. Using the transformations in Equation $(2.12), \theta_{m, \mu}(\tau, z)$ can be shown to transform diagonally under $\Gamma(4 m)^{*}, 4$

$$
\left.\theta_{m, \mu}\right|_{\frac{1}{2}, m} \gamma=\theta_{m, \mu}(\tau, z), \quad \gamma \in \Gamma(4 m)^{*} .
$$

Note that $\theta_{m, \mu}(\tau, z)$ is not multiplied by an additional unitary pre-factor; $\mathbf{M}_{2 m}(\gamma)$ is thus indeed the identity matrix for $\gamma \in \Gamma(4 m)^{*}$. More general transformations, acting diagonally on $\theta_{m, \mu}(\tau, z)$ but with non-trivial unitary pre-factor, form a larger congruence subgroup.

From the above considerations, we deduce that the matrices $\mathbf{M}_{2 m}(\gamma)$ form a $2 m$-dimensional representation of the finite group $\tilde{\Gamma} / \Gamma(4 m)^{*}$. Since the generators of $\tilde{\Gamma}, S$ and $T$, are both represented by unitary matrices $\mathbf{M}_{2 m}(S)$ and $\mathbf{M}_{2 m}(T)$, the representation is unitary.

The transformations of the theta functions combined with those of $\phi(\tau, z)$ (given by $(2.2)$ in terms of $Z(\tau, z)$ ) determine that the functions $h_{\mu}(\tau)$ transform as a vector-valued modular form with weight $-\frac{1}{2}$, and conjugately to the transformations of $\theta_{m, \mu}$,

$$
\left(\begin{array}{c}
\left.h_{-m+1}\right|_{-\frac{1}{2}} \gamma \\
\left.h_{-m+2}\right|_{-\frac{1}{2}} \gamma \\
\ldots \\
\ldots \\
\left.h_{m}\right|_{-\frac{1}{2}} \gamma
\end{array}\right)=\mathbf{M}_{2 m}^{-1}(\gamma)\left(\begin{array}{c}
h_{-m+1} \\
h_{-m+2} \\
\ldots \\
\ldots \\
h_{m}
\end{array}\right)
$$

for $\gamma \in \tilde{\Gamma}$. Since the representation is unitary, $\mathbf{M}_{2 m}^{-1}(\gamma)=\overline{\mathbf{M}_{2 m}(\gamma)}$.

The modular forms $h_{\mu}(\tau), \mu \in \mathbb{Z} / 2 m \mathbb{Z}$, are not all linearly independent forms. This is a consequence of the fact that $(-1,1)$ acts non-trivially on $\theta_{m, \mu}(\tau, z)$ but leaves $h_{\mu}(\tau)$ invariant. The equality $\left.\phi\right|_{0, m}(-1,1)=\phi$, which is equivalent to

$$
\phi(\tau, z)=\phi(\tau,-z),
$$

requires then that $h_{-\mu}(\tau)=h_{\mu}(\tau)$. The modular transformations of the vector-valued modular form $h_{\mu}(\tau)$ are therefore adequately described by a vector of length $m+1$ and an $(m+1) \times(m+1)$ matrix $\overline{\mathbf{M}(\gamma)}$. This gives rise to an $(m+1)$-dimensional representation of the finite group $\tilde{\Gamma} / \Gamma(4 m)^{*}$. The domain of $\mu$ in this representation is taken to be $[0, m]$.

\footnotetext{
${ }^{4}$ In the following, the tilde is omitted from elements $\tilde{\gamma} \in \tilde{\Gamma}$.
} 


\section{Cusp forms as constraints on $\mathcal{N}=(2,2)$ spectra}

This section calculates the number of independent constraints on the polar spectrum, which is the number of constraints on the polar coefficients $c_{\mu}(n)$, $n<0$, of a Jacobi form. This result, given in (3.19), allows us to determine the dimension $\tilde{J}_{0, m}$ or equivalently the space of elliptic genera in (3.22).

Before considering the constraints, it is first shown that the weakly holomorphic, negative weight modular form $h_{\mu}(\tau)$ in (2.5) and (2.6) is uniquely determined by its polar coefficients. One would namely find a contradiction when two or more such forms exist, having the same principal part but different regular part. The difference of two such forms would be a holomorphic, negative weight modular form of $\Gamma(4 m)^{*}$. Since such holomorphic forms with negative weight do not exist, the polar coefficients uniquely determine the weakly holomorphic modular form. The upper bound on the dimension of weakly holomorphic, negative weight modular forms, with a given maximum order of the pole at $\tau \rightarrow i \infty$, is therefore given by the number of polar terms $p(m)$.

One encounters the presence of constraints when one attempts to find a negative weight modular form with a prescribed set of polar coefficients $[11,12]$. Naively, a sum over $\Gamma / \Gamma_{\infty}$, which is known as a Poincaré series, completes a function which is not modular covariant to a modular covariant object. However, Manschot and Moore [11] and Niebur [12] explain that a sum over $\Gamma / \Gamma_{\infty}$ does not complete every possible choice of polar terms to a modular form or Jacobi form. Manschot and Moore [11] aim to construct a Jacobi form by a Poincaré series on the principal part of $h_{\mu}(\tau)$. For a general choice of $c_{\mu}(n), n<0$, the constructed function $\phi(\tau, z)$ does not transform as a form in $\tilde{J}_{0, m}$. Instead, $\phi(\tau, z)$ transforms with an anomalous shift under $\Gamma$-transformations

$$
\left.\phi\right|_{0, m} \gamma-\phi=-\frac{1}{\Gamma(3 / 2)} \sum_{\mu} \theta_{m, \mu}(\tau, z) \int_{\gamma^{-1}(\infty)}^{-i \infty} \overline{g_{\mu}(t)}(\bar{t}-\tau)^{1 / 2} d \bar{t}
$$

where $\Gamma(x)$ is the Gamma-function. The vector $g_{\mu}(\tau)$ is a vector-valued cusp form of weight $2 \frac{1}{2}$ and it transforms conjugately to $h_{\mu}(\tau)$, thus with the $(m+$ $1) \times(m+1)$ matrices $\mathbf{M}(\gamma)$. We refer to the space of these vector-valued cusp forms as $S_{2 \frac{1}{2}, \mathbf{M}}\left(\Gamma(4 m)^{*}\right) ; S_{2 \frac{1}{2}, \mathbf{M}}\left(\Gamma(4 m)^{*}\right)$ has the argument $\Gamma(4 m)^{*}$ since the matrices $\mathbf{M}(\gamma)$ form a representation of $\tilde{\Gamma} / \Gamma(4 m)^{*}$. The righthand side does not vanish unless $g_{\mu}(\tau)=0$ [12]. Moreover, independent vector-valued forms $g_{\mu}(\tau)$ lead to independent vector-valued functions of $\tau[22]$ after the integration over $\bar{t}$ in (3.1). 
The cusp form $g_{\mu}(\tau)$ is a Poincaré series determined by the coefficients $c_{\mu}(n), n<0[11,12]$. Vanishing of $g_{\mu}(\tau)$ is established for a proper choice of $c_{\mu}(n), n<0,{ }^{5}$ namely a choice which corresponds to the coefficients of a weak Jacobi form. To learn whether a given polar spectrum is consistent with the space of potential elliptic genera, one can check whether the corresponding cusp form vanishes or not. Since the Poincaré series span the space of cusp forms [24] and the integration leads to independent functions of $\tau$, the number of independent constraints is equal to the dimension of the space of vector-valued cusp forms. More details can be found in [11]. In the context of scalar modular forms, Niebur [12] established a space of cusp forms as an obstruction to the construction of non-positive weight modular forms with singularities.

The space of cusp forms forms thus an obstruction to the construction of Jacobi forms and imposes restrictions on the choice of polar coefficients. The space $P(m)$ reduced by the constraints is denoted by $P_{\mathrm{c}}(m)$. Since a specific choice of polar coefficients which lies in $P_{\mathrm{c}}(m)$ corresponds to a unique form in $\tilde{J}_{0, m}, \operatorname{dim} P_{\mathrm{c}}(m)$ is equal to $\operatorname{dim} \tilde{J}_{0, m}$. This dimension can thus be calculated by

$$
\operatorname{dim} \tilde{J}_{0, m}=p(m)-\operatorname{dim} S_{2 \frac{1}{2}, \mathbf{M}}\left(\Gamma(4 m)^{*}\right) .
$$

The different spaces and the described relations between them can be nicely summarized as a short exact sequence

$$
0 \rightarrow \tilde{J}_{0, m} \rightarrow P(m) \rightarrow S_{2 \frac{1}{2}, \mathbf{M}}\left(\Gamma(4 m)^{*}\right) \rightarrow 0,
$$

where the second arrow maps a given Jacobi form to the set of polar coefficients $c_{\mu}(n), n<0$, and the third arrow is the construction of the vectorvalued cusp form from the polar coefficients by a Poincaré series [11].

The obstructions can be viewed as a manifestation of the Mittag-Leffler problem [25], which is the problem of finding a meromorphic section with prescribed singularities of a line bundle $\mathcal{L}$ over a manifold $X$. The space of obstructions to finding such a section is given by $H^{1}(X, \mathcal{O}(\mathcal{L}))$, where $\mathcal{O}(\mathcal{L})$ is the sheaf of holomorphic sections of $\mathcal{L}$. Since the modular curve $\mathcal{H} / \Gamma$ is onedimensional, $H^{1}(X, \mathcal{O}(\mathcal{L}))$ is by Serre duality related to $H^{0}\left(X, \mathcal{O}\left(K \times \mathcal{L}^{*}\right)\right)$, with $K$ the canonical bundle. In the present discussion, sections of $\mathcal{L}$ have weight $-\frac{1}{2}$ and therefore sections of $\mathcal{L}^{*}$ have weight $\frac{1}{2}$. Since holomorphic

\footnotetext{
${ }^{5}$ In the case of bosonic pure gravity [23], the corresponding cusp form would be a weight 2 cusp form of $\Gamma$. Since these do not exist, no constraints by modularity are imposed on the polar spectrum, which is consistent with [23].
} 
sections of $K$ are cusp forms of weight 2, this explains the appearance of cusp forms of weight $2 \frac{1}{2}$ as obstructions to the construction of elliptic genera. Borcherds [26] generalizes these considerations to the vector-valued case and proves that the obstruction space for vector-valued modular forms $h_{\mu}(\tau)$ is given by the vector-valued cusp forms $g_{\mu}(\tau)$. Explicit calculations of the dimensions of vector-valued modular forms are carried out in [15]. Such dimension formulas are also mentioned in [26-28].

The basic ingredients for the calculation of $\operatorname{dim} S_{2 \frac{1}{2}, \mathbf{M}}\left(\Gamma(4 m)^{*}\right)$ are the orthogonality relations for irreducible characters of finite groups and the Selberg trace formula. The relevant finite group appeared in Section 2, namely $\tilde{\Gamma} / \Gamma(4 m)^{*}$. The transformation properties of $g_{\mu}(\tau)$ provide an $(m+$ 1)-dimensional representation $\mathbf{M}$ in terms of the matrices $\mathbf{M}(\gamma)$. We define a character of this representation in the usual way by

$$
\chi_{\mathbf{M}}(\gamma)=\operatorname{Tr}(\mathbf{M}(\gamma))
$$

We label the set of irreducible representations by $\mathbf{R}_{i}$. The orthogonality relations for characters of finite groups read in this case

$$
\frac{1}{\left|\tilde{\Gamma} / \Gamma(4 m)^{*}\right|} \sum_{\gamma \in \tilde{\Gamma} / \Gamma(4 m)^{*}} \chi_{\mathbf{R}_{i}}(\gamma) \overline{\chi_{\mathbf{R}_{j}}(\gamma)}=\delta_{i j}
$$

The multiplicities $m_{i}$ of the irreducible representations $\mathbf{R}_{i}$ in $\mathbf{M}$ are given by

$$
m_{i}=\frac{1}{\left|\tilde{\Gamma} / \Gamma(4 m)^{*}\right|} \sum_{\gamma \in \tilde{\Gamma} / \Gamma(4 m)^{*}} \chi_{\mathbf{M}}(\gamma) \overline{\chi_{\mathbf{R}_{j}}(\gamma)}
$$

Since $\Gamma(4 m)^{*}$ lies in the kernel of the representation $\mathbf{M}$, the individual vector elements $g_{\mu}(\tau)$ lie in the space of weight $2 \frac{1}{2}$ cusp forms of $\Gamma(4 m)^{*}$, which is denoted by $S_{2 \frac{1}{2}}\left(\Gamma(4 m)^{*}\right)$. Since $\Gamma(4 m)^{*}$ is a normal subgroup of $\tilde{\Gamma}$, the space $S_{2 \frac{1}{2}}\left(\Gamma(4 m)^{*}\right)$ is closed under transformations of $\gamma \in \tilde{\Gamma}$; such transformations rotate a chosen set of basis elements of $S_{2 \frac{1}{2}}\left(\Gamma(4 m)^{*}\right)$ among each other. As a consequence, $S_{2 \frac{1}{2}}\left(\Gamma(4 m)^{*}\right)$ defines a $\operatorname{dim}_{2 \frac{1}{2}}\left(\Gamma(4 m)^{*}\right)$-dimensional representation of $\tilde{\Gamma} / \Gamma(4 m)^{*}$, which can similarly be decomposed into the irreducible representations. When the multiplicities of $\mathbf{R}_{i}$ in $S_{2 \frac{1}{2}}\left(\Gamma(4 m)^{*}\right)$ are $s_{i}$, then $\operatorname{dim} S_{2 \frac{1}{2}, \mathbf{M}}\left(\Gamma(4 m)^{*}\right)=\sum_{i} m_{i} s_{i}$. The character of the element $\gamma$ in the $\tilde{\Gamma} / \Gamma(4 m)^{*}$-representation $S_{2 \frac{1}{2}}\left(\Gamma(4 m)^{*}\right)$ is denoted by

$$
\operatorname{Tr}\left[\gamma, S_{2 \frac{1}{2}}\left(\Gamma(4 m)^{*}\right)\right]
$$


In terms of the characters, $\operatorname{dim} S_{2 \frac{1}{2}, \mathbf{M}}\left(\Gamma(4 m)^{*}\right)$ is now expressed by (3.8)

$$
\operatorname{dim} S_{2 \frac{1}{2}, \mathbf{M}}\left(\Gamma(4 m)^{*}\right)=\frac{1}{\left|\tilde{\Gamma} / \Gamma(4 m)^{*}\right|} \sum_{\gamma \in \tilde{\Gamma} / \Gamma(4 m)^{*}} \chi_{\mathbf{M}}(\gamma) \overline{\operatorname{Tr}\left[\gamma, S_{2 \frac{1}{2}}\left(\Gamma(4 m)^{*}\right)\right]} .
$$

The Selberg trace formula provides a way to determine traces as in Equation (3.7), for example, $\operatorname{Tr}\left[\gamma, S_{w}\left(\Gamma(4 m)^{*}\right)\right]-\operatorname{Tr}\left[\gamma^{-1}, M_{2-w}\left(\Gamma(4 m)^{*}\right)\right]$ can be calculated. The space $M_{2-w}\left(\Gamma(4 m)^{*}\right)$ is the space of holomorphic modular forms of $\Gamma(4 m)^{*}$ with weight $2-w$. This is applied by Theorem 5.1 of [15] to calculate the difference of the dimensions of $S_{w, \mathbf{M}}\left(\Gamma(4 m)^{*}\right)$ and $M_{2-w, \overline{\mathbf{M}}}\left(\Gamma(4 m)^{*}\right)$, where $\mathbf{M}$ is a representation of $\tilde{\Gamma} / \Gamma(4 m)^{*}$. It is a sum of three (generically fractional) contributions

$$
\operatorname{dim} S_{w, \mathbf{M}}\left(\Gamma(4 m)^{*}\right)-\operatorname{dim} M_{2-w, \overline{\mathbf{M}}}\left(\Gamma(4 m)^{*}\right)=A_{\mathrm{s}}+A_{\mathrm{e}}+A_{\mathrm{p}}
$$

The subscripts "s," "e" and "p" refer respectively to "scalar," "elliptic" and "parabolic." This terminology appears naturally in the derivation of the Selberg trace formula, see for example, [29]. The three contributions are given in $[15,27]$

$$
\begin{aligned}
& A_{\mathrm{s}}=\frac{w-1}{12} \chi_{\mathbf{M}}(1), \\
& A_{\mathrm{e}}=\frac{1}{4} \operatorname{Re}\left[e\left(\frac{w}{4}\right) \chi_{\mathbf{M}}(S)\right]+\frac{2}{3 \sqrt{3}} \operatorname{Re}\left[e\left(\frac{2 w+1}{12}\right) \chi_{\mathbf{M}}(S T)\right], \\
& A_{\mathrm{p}}=-\frac{1}{2} S(\mathbf{M})-\sum_{j=1}^{d}\left(\left(\lambda_{j}\right)\right),
\end{aligned}
$$

where the trace of the identity matrix $\chi_{\mathbf{M}}(1)$ is the dimension $d$ of the representation $\mathbf{M}$. The numbers $\lambda_{j}$ are the fractional numbers appearing in $\chi_{\mathbf{M}}\left(T^{n}\right)=\sum_{j} e\left(\lambda_{j} n\right)$. The symbol $S(\mathbf{M})$ is defined as the number of $\lambda_{j}$ which take values in $\mathbb{Z}$. The function $((x))$ is defined by

$$
((x))=x-\frac{\lceil x\rceil+\lfloor x\rfloor}{2}= \begin{cases}\xi-\frac{1}{2}, & \text { if } x=\xi+\mathbb{Z}, 0<\xi<1, \\ 0, & \text { if } x \in \mathbb{Z} .\end{cases}
$$

Equation (3.9) provides us $\operatorname{dim} S_{2 \frac{1}{2}, \mathbf{M}}\left(\Gamma(4 m)^{*}\right)$, since holomorphic modular forms with negative weight do not exist and hence $\operatorname{dim} M_{-\frac{1}{2}, \overline{\mathbf{M}}}$ $\left(\Gamma(4 m)^{*}\right)=0$. To calculate the dimension of $S_{2 \frac{1}{2}, \mathbf{M}}\left(\Gamma(4 m)^{*}\right)$, one needs 
to evaluate $\chi_{\mathbf{M}}(\gamma)$ for the relevant $\gamma^{\prime}$ s and substitute in Equation (3.10). We proceed with a direct evaluation of $\chi_{\mathbf{M}}(\gamma)$ from the matrices defined in Section 2. From Equation (2.18) we deduced that the $h_{\mu}(\tau)$ are described by an $(m+1)$-dimensional vector, thus $d=m+1$ and $\mu=0, \ldots, m$. One can express $\left.g_{\mu}\right|_{2 \frac{1}{2}} S$ and $\left.g_{\mu}\right|_{2 \frac{1}{2}} T$ in terms of $g_{\mu}(\tau)$ with $\mu=0, \ldots, m$ :

$$
\begin{aligned}
\left.g_{\mu}\right|_{2 \frac{1}{2}} S= & \frac{1}{\sqrt{2 m i}}\left(g_{0}(\tau)-e\left(\frac{\mu}{2}\right) g_{m}(\tau)\right. \\
& \left.+\sum_{\nu=1}^{m}\left[e\left(\frac{\mu \nu}{2 m}\right)+e\left(-\frac{\mu \nu}{2 m}\right)\right] g_{\nu}(\tau)\right) \\
\left.g_{\mu}\right|_{2 \frac{1}{2}} T= & e\left(\frac{\mu^{2}}{4 m}\right) g_{\mu}(\tau) .
\end{aligned}
$$

$A_{\mathrm{s}}$ and $A_{\mathrm{p}}$ can straightforwardly be determined to be:

$$
\begin{aligned}
& A_{\mathrm{s}}=\frac{m+1}{8} \\
& A_{\mathrm{p}}=-\frac{1}{2} S(m)-\sum_{\nu=0}^{m}\left(\left(\frac{\nu^{2}}{4 m}\right)\right),
\end{aligned}
$$

where $S(m)$ is equal to the number of times $\frac{\nu^{2}}{4 m} \in \mathbb{Z}$ for $\nu \in[0, m]$, which can be shown to be equal to $\left\lfloor\frac{b+2}{2}\right\rfloor$, with $b$ the largest integer whose square divides $m$ and $\lfloor\cdot\rfloor$ the floor function. The sum over $\nu$ in Equation (3.14) can be related to a sum over class numbers [14], which is more convenient when one wants to evaluate the sum for large $m$.

We evaluate now $A_{\mathrm{s}}$. By Equation (3.12), we get

$$
\begin{aligned}
\chi_{\mathbf{M}}(S) & =\frac{1}{\sqrt{2 m i}}\left(1-e\left(\frac{m}{2}\right)+\sum_{\mu=1}^{m} e\left(\frac{\mu^{2}}{2 m}\right)+e\left(-\frac{\mu^{2}}{2 m}\right)\right), \\
\chi_{\mathbf{M}}(S T) & =\frac{1}{\sqrt{2 m i}}\left(1-e\left(\frac{3 m}{4}\right)+\sum_{\mu=1}^{m} e\left(\frac{3 \mu^{2}}{4 m}\right)+e\left(-\frac{\mu^{2}}{4 m}\right)\right) .
\end{aligned}
$$


These sums can be calculated using the analysis of quadratic Gauss sums $G(n, m)=\sum_{r=1}^{m} e\left(\frac{n r^{2}}{m}\right)[30]$. The relevant Gauss sums in this case are

$$
\begin{aligned}
& G(1,2 m)=\left\{\begin{array}{lll}
\sqrt{2 m}(1+i), & m=0 & \bmod 2, \\
0, & m=1 & \bmod 2,
\end{array}\right. \\
& G(3,4 m)=\left\{\begin{array}{lll}
2 \sqrt{3 m}(1+i), & m=0 & \bmod 3, \\
2 \sqrt{m}(1-i), & m=1 & \bmod 3, \\
-2 \sqrt{m}(1-i), & m=2 & \bmod 3 .
\end{array}\right.
\end{aligned}
$$

Then we obtain for $\chi_{\mathbf{M}}(S)$ and $\chi_{\mathbf{M}}(S T)$

$$
\begin{gathered}
\chi_{\mathbf{M}}(S)=\left\{\begin{array}{lll}
e\left(-\frac{1}{8}\right), & m=0 & \bmod 2, \\
0, & m=1 & \bmod 2,
\end{array}\right. \\
\chi_{\mathbf{M}}(S T)=\left\{\begin{array}{lll}
e\left(-\frac{1}{12}\right), & m=0 & \bmod 3, \\
e\left(-\frac{1}{4}\right), & m=1 & \bmod 3, \\
0, & m=2 & \bmod 3 .
\end{array}\right.
\end{gathered}
$$

Inserting this in Equation (3.10) with $w=2 \frac{1}{2}$, we find

$$
A_{\mathrm{e}}=\left\{\begin{array}{lll}
-\frac{1}{4}, & m=0 & \bmod 2, \\
0, & m=1 & \bmod 2,
\end{array}+ \begin{cases}-\frac{1}{3}, & m=0 \quad \bmod 3, \\
0, & m=1,2 \bmod 3 .\end{cases}\right.
$$

Thus, our final result for $\operatorname{dim} S_{2 \frac{1}{2}, \mathbf{M}}\left(\Gamma(4 m)^{*}\right)$ is

$$
\begin{aligned}
& \operatorname{dim} S_{2 \frac{1}{2}, \mathbf{M}}\left(\Gamma(4 m)^{*}\right)=\frac{m+1}{8}-\frac{1}{2} S(m)-\sum_{\nu=0}^{m}\left(\left(\frac{\nu^{2}}{4 m}\right)\right) \\
& +\left\{\begin{array}{lll}
-\frac{1}{4}, & m=0 & \bmod 2, \\
0, & m=1 & \bmod 2,
\end{array}+ \begin{cases}-\frac{1}{3}, & m=0 \bmod 3, \\
0, & m=1,2 \bmod 3 .\end{cases} \right.
\end{aligned}
$$

The right-hand side grows linearly with $m$ for large $m$, it acquires its first non-vanishing value for $m=5$. The quantities $A_{\mathrm{s}}, A_{\mathrm{e}}$ and $A_{\mathrm{p}}$ behave differently when $m$ is increased. Equation (3.13) shows that $A_{\mathrm{s}}$ grows linearly with $m$ and $A_{\mathrm{p}}$ as $\sqrt{m}$. The absolute value of $A_{\mathrm{e}}$ is always $<1$ and completes $A_{\mathrm{s}}+A_{\mathrm{p}}$, such that the total sum is an integer. 
Skoruppa [15] evaluates $\chi_{\mathbf{M}}(\gamma)$ by a decomposition of the representation $\mathbf{M}$ into irreducible representations, and an evaluation of the characters of the irreducible representations. The final dimension formulas are rather intricate and involve several sums over integers and arithmetic functions. The values obtained for $\operatorname{dim} S_{2 \frac{1}{2}, \mathrm{M}}\left(\Gamma(4 m)^{*}\right), m=1, \ldots, 14$, by these formulas are identical to those obtained by Equation (3.19). The equivalence is however not proven for general $m$.

The dimension of the space of elliptic genera or weak Jacobi forms is given by the number of polar coefficients $p(m)$ minus the number of constraints (3.22). The number $p(m)$ can be calculated as a function of $m$ [14]

$$
p(m)=\sum_{\nu=0}^{m}\left\lceil\frac{\nu^{2}}{4 m}\right\rceil
$$

The evaluation of $p(m)$ is less elaborate when the sum over $\nu$ is rewritten using the functions $S(m)$ and $((x))$ introduced in Equation (3.14). This gives for $p(m)[14]$

$$
\begin{aligned}
p(m) & =\sum_{\nu=0}^{m}\left\{\frac{\nu^{2}}{4 m}+\frac{1}{2}-\left(\left(\frac{\nu^{2}}{4 m}\right)\right)\right\}-\frac{1}{2} S(m), \\
& =\frac{m^{2}}{12}+\frac{5 m}{8}+\frac{13}{24}-\frac{1}{2} S(m)-\sum_{\nu=0}^{m}\left(\left(\frac{\nu^{2}}{4 m}\right)\right) .
\end{aligned}
$$

A comparison with (3.19) shows that the ratio of $p(m)$ and the number of constraints grows linearly with the central charge.

The number of polar coefficients and the constraints on the polar spectrum are listed in Table 1. A general expression for $\operatorname{dim} \tilde{J}_{0, m}$ is obtained by

Table 1: The number of polar coefficients $p(m)$ and the number of constraints on these coefficients, $\operatorname{dim} S_{2 \frac{1}{2}, \mathbf{M}}\left(\Gamma(4 m)^{*}\right)$, for $\mathcal{N}=(2,2)$ elliptic genera as a function of $m$.

\begin{tabular}{lrrrrrrrrrrrrrr}
\hline$m$ & 1 & 2 & 3 & 4 & 5 & 6 & 7 & 8 & 9 & 10 & 11 & 12 & 13 & 14 \\
$p(m)$ & 1 & 2 & 3 & 4 & 6 & 8 & 9 & 11 & 13 & 16 & 18 & 21 & 23 & 27 \\
$\operatorname{dim} S_{2 \frac{1}{2}, \mathbf{M}}\left(\Gamma(4 m)^{*}\right)$ & 0 & 0 & 0 & 0 & 1 & 1 & 1 & 1 & 1 & 2 & 2 & 2 & 2 & 3 \\
\hline
\end{tabular}


inserting Equations (3.19) and (3.21) in (3.2),

$$
\begin{aligned}
\operatorname{dim} \tilde{J}_{0, m}= & \frac{m^{2}}{12}+\frac{m}{2}+\frac{5}{12}+\left\{\begin{array}{lll}
\frac{1}{4}, & m=0 & \bmod 2 \\
0, & m=1 & \bmod 2,
\end{array}\right. \\
& + \begin{cases}\frac{1}{3}, & m=0 \quad \bmod 3, \\
0, & m=1,2 \quad \bmod 3 .\end{cases}
\end{aligned}
$$

This result is identical to the dimension formula calculated in [14]. There, the dimension formula is derived by a study of the Taylor expansion in $z$ of $\phi(\tau, z)$. This approach determines that $\operatorname{dim} \tilde{J}_{0, m}$ is equal to

$$
\operatorname{dim} \tilde{J}_{0, m}=\sum_{\nu=0}^{m} \operatorname{dim} M_{2 \nu}(\Gamma)
$$

where $M_{2 \nu}(\Gamma)$ is the space of modular forms of weight $2 \nu$. The dimension of $M_{2 \nu}(\Gamma)$ is given by [14]

$$
\operatorname{dim} M_{2 \nu}=\frac{2 \nu+5}{12}-\frac{1}{3} \chi_{3}(2 \nu-1)-\frac{1}{4} \chi_{4}(2 \nu-1),
$$

where $\chi_{3}$ and $\chi_{4}$ are the non-trivial Dirichlet characters modulo 3 and 4 . Substitution of this expression in Equation (3.23) and evaluation of the sum over $\nu$ results precisely in Equation (3.22) [14].

The technique to calculate $\operatorname{dim} \widetilde{J}_{0, m}$, described in the previous sections can be easily generalized to other spaces of Jacobi forms. For example, one can determine the dimensions of spaces of Jacobi (cusp) forms with weight $k \geq 3$, by requiring the vector $h_{\mu}(\tau)$ to be a holomorphic (cusp) form with weight $k-\frac{1}{2}$. The described techniques let us calculate the dimension of the appropriate spaces. Dimensions of such Jacobi forms can also be obtained by a trace formula for Jacobi forms [31-33] or the Riemann-Roch theorem [34].

Another generalization is the calculation of dimensions of spaces of weak Jacobi forms with general weight, $\operatorname{dim} \tilde{J}_{k, m}$. This might be relevant for physics, for example, in the context of modular differential equations for superconformal characters [35]. Here we concentrate on $k$ even. Generically, the polar coefficients might not determine the vector-valued form $h_{\mu}(\tau)$ uniquely. When the weight is positive, $k-\frac{1}{2}>0$, holomorphic forms might exist and some non-polar terms must be specified as well to fix the $h_{\mu}(\tau)$. The number of these coefficients is given by $\operatorname{dim} M_{k-\frac{1}{2}, \overline{\mathbf{M}}}\left(\Gamma(4 m)^{*}\right)$. Of course, a number of obstructions to finding $h_{\mu}(\tau)$ might still exist. This 
number is given by $\operatorname{dim} S_{2 \frac{1}{2}-k, \mathbf{M}}\left(\Gamma(4 m)^{*}\right)$. As a result, $\operatorname{dim} \tilde{J}_{k, m}$ is still given by the Selberg trace formula

$$
\operatorname{dim} \tilde{J}_{k, m}=p(m)-\left(\operatorname{dim} S_{2 \frac{1}{2}-k, \mathbf{M}}\left(\Gamma(4 m)^{*}\right)-\operatorname{dim} M_{k-\frac{1}{2}, \overline{\mathbf{M}}}\left(\Gamma(4 m)^{*}\right)\right) .
$$

Using the equations in Section 3, one can readily evaluate the right-hand side of (3.25). One finds

$$
\begin{aligned}
& \operatorname{dim} \tilde{J}_{k, m}=\frac{m^{2}}{12}+\frac{5 m}{8}+\frac{13}{24}-\frac{3-2 k}{24}(m+1) \\
& -\frac{1}{4}\left\{\begin{array}{llll}
-1, & m=0 & \bmod 2, k=0 & \bmod 4, \\
1, & m=0 & \bmod 2, k=2 & \bmod 4, \\
0, & \text { else, } & &
\end{array}\right. \\
& -\frac{1}{3}\left\{\begin{array}{llll}
-1, & m=0 & \bmod 3, k=0 & \bmod 6, \\
1, & m=0 & \bmod 3, k=2 & \bmod 6, \\
1, & m=1 & \bmod 3, k=2 & \bmod 6, \\
-1, & m=1 & \bmod 3, k=4 & \bmod 6, \\
0, & \text { else. } & &
\end{array}\right.
\end{aligned}
$$

This is in agreement with the formulas given in [14]. Note that in the case of $k=2$, both $\operatorname{dim} S_{2 \frac{1}{2}-k, \mathbf{M}}\left(\Gamma(4 m)^{*}\right)$ and $\operatorname{dim} M_{k-\frac{1}{2}, \overline{\mathbf{M}}}\left(\Gamma(4 m)^{*}\right)$ might be nonzero. The dimension formula for $\operatorname{dim} \tilde{J}_{2, m}$ does not provide us the number of polar and non-polar coefficients which need to be fixed.

\section{Constraints on $\mathcal{N}=(4,0)$ spectra}

This section considers the constraints on the polar spectrum of $\mathcal{N}=(4,0)$ SCFTs by the modular symmetries of the $\mathcal{N}=(4,0)$ elliptic genus. The physical properties of this SCFT determine that the elliptic genus is a mild generalization of a Jacobi form. As in Section 2, a quasi-periodicity property can be derived from a spectral flow symmetry, leading to a theta function decomposition. The theta functions involved are however sums over a higher dimensional, non-definite, integral lattice $\Lambda$, which leads to a vector $z^{a}$ of elliptic variables. The representation of $\tilde{\Gamma} / \Gamma(4 m)^{*}$ associated with the vector-valued modular forms is also more intricate.

The constraints are determined by the technique outlined in the previous sections for weak Jacobi forms. The Taylor expansion, which is used in [14] to determine the dimension formulas, is not useful in this context 
for several reasons. The main complications are that the theta functions generically do not cancel the poles of the vector-valued modular forms, the higher dimensional lattice $\Lambda$, and that the $\mathcal{N}=(4,0)$ elliptic genus depends on $\tau$ as well as $\bar{\tau}$.

As a final example of the calculation of constraints, the application to the partition functions of $\mathcal{N}=4$ topologically twisted Yang-Mills on 4-manifolds is discussed [36]. The case of $\mathbb{C P}^{2}$ is worked out in some detail. The modular properties of the vector-valued modular form, obtained from the $(4,0)$ elliptic genus, closely resembles the properties of the set of gauge theory partition functions for the groups $\mathrm{SU}(N) / \mathbb{Z}_{N}$.

The following paragraphs explain the appearance and relevance of these generalizations of Jacobi forms in physics. Readers who are only interested in the mathematical side of the discussion might want to go directly to Equation (4.7).

The main physical relevance of $\mathcal{N}=(4,0)$ elliptic genera is the fact that the degeneracies of M-theory black holes are enumerated by such partition functions $[7,16-19]$. Knowledge of the constraints on the polar degeneracies is useful when one wants to determine explicit expressions for such $\mathcal{N}=$ $(4,0)$ elliptic genera, as is done in $[17,37]$. Denef and Moore [38] derive an identical partition function from the viewpoint of IIA string theory. The $\mathcal{N}=(4,0)$ SCFT arises as the boundary CFT in the near-horizon geometry of an M-theory black hole. The near-horizon geometry is given by $\mathrm{AdS}_{3} \otimes$ $S^{2} \otimes X$, where $X$ is a six-dimensional Calabi-Yau. The black hole is sourced by an M5-brane with world-volume fluxes, which wraps an ample divisor in $X$ and the boundary torus $T^{2}$ of $\mathrm{AdS}_{3}$. The Poincare dual of the divisor is $P=p^{a} \alpha_{a}$; the $\alpha_{a}$ 's form a basis of $H^{2}(X, \mathbb{Z})$ with $a=1, \ldots, b_{2}$. The SCFT is obtained as a reduction of the six-dimensional low-energy degrees of freedom to $T^{2}$. To this end, the typical length scale of the torus is required to be much larger than that of $X$, and the M5-branes must be scarce in $X$ to avoid gravitational effects. Due to the amount of supersymmetry, the trace over the Ramond sector needs an insertion of $F^{2}$ to be non-vanishing [39]. The $\mathcal{N}=(4,0)$ elliptic genus $Z(\tau, \bar{\tau}, z)$ is defined as

$$
Z(\tau, \bar{\tau}, z)=\operatorname{Tr}_{\mathrm{R}} \frac{1}{2} F^{2}(-1)^{F+p \cdot J_{0}} q^{L_{0}-c_{L} / 24} \bar{q}^{\bar{L}_{0}-c_{R} / 24} y^{J_{0}},
$$

where $y^{J_{0}}=e\left(z \cdot J_{0}\right)$. The operators $J_{0, a}$ are the generators of $b_{2} U(1)$ charges. The charges are denoted by $q_{a}$ and represent the M2-brane charge of the black hole. An electric charge vector $q$ is valued in the shifted dual lattice $\Lambda^{*}+p / 2$, where $\Lambda^{*}$ is the dual lattice of the magnetic charge lattice $\Lambda$. The shift is a consequence of the Freed-Witten anomaly [40]. An element $k \in \Lambda$ 
is an element of $H^{2}(X, \mathbb{Z})$. The pull back of the inclusion map $i: P \hookrightarrow X$ provides a non-degenerate integral inner-product $D$ on $H^{2}(X, \mathbb{Z})$

$$
D: H^{2}(X, \mathbb{Z}) \otimes H^{2}(X, \mathbb{Z}) \rightarrow \mathbb{C}, \quad D(\rho, \sigma)=\int_{P} i^{*} \rho \wedge i^{*} \sigma=\int_{X} \rho \wedge \sigma \wedge P .
$$

The quadratic form written as a matrix is $d_{a b}=d_{a b c} p^{c}$, where $d_{a b c}=\int_{X} \alpha_{a} \wedge$ $\alpha_{b} \wedge \alpha_{c}$ is the triple intersection number of four-cycles. The inner product on the dual lattice is $d^{a b}=\left(d_{a b}\right)^{-1}$. The dimension of this lattice is the second Betti number $b_{2}$ and the signature is $\left(1, b_{2}-1\right)$.

The central charges $c_{L}$ and $c_{R}$ of the SCFT are given by

$$
c_{L}=p^{3}+\frac{1}{2} c_{2} \cdot p, \quad c_{R}=p^{3}+c_{2} \cdot p,
$$

where $c_{2}$ is the second Chern class of $X$. The zero-point energies and the central charges determine that the momentum on the torus can take the following values

$$
L_{0}-\bar{L}_{0}-\frac{c_{L}-c_{R}}{24}=\frac{p^{3}}{8}+\frac{c_{R}}{24} \bmod \mathbb{Z}
$$

We denote the left-hand side of this equation by $-q_{\overline{0}}$, where $q_{\overline{0}}$ is the antiD0-brane charge in IIA string theory. The quantity $q_{\overline{0}}+\frac{1}{2} q^{2}$ is positive for black holes. The charges $p^{a}$ and $q_{a}$ correspond, respectively, to the D4-brane and D2-brane charges in IIA string theory. The insertion of $F^{2}$ projects the trace on $\frac{1}{2}$-BPS states. For these states, $L_{0}-\frac{c_{L}}{24}$ satisfies

$$
L_{0}-\frac{c_{L}}{24}=\frac{1}{2} q_{+}^{2}
$$

where the subscript + denotes projection to the positive definite sublattice. This condition implies a "heat equation"

$$
\left(\partial_{\tau}+\frac{i}{4 \pi} \partial_{z_{+}}^{2}\right) Z(\tau, \bar{\tau}, z)=0
$$

The weight of $Z(\tau, \bar{\tau}, z)$ is $\left(\frac{1}{2},-\frac{3}{2}\right)$, which is a consequence of the spacetime momenta and the insertion of $F^{2}$. Since the quantity in Equation (4.4) is in general not an integer, $\Gamma$-transformations are accompanied by phase factors

$$
\begin{aligned}
& \left.Z\right|_{(1 / 2,-3 / 2)} S=\varepsilon(S) e\left(\frac{z_{+}^{2}}{2 \tau}+\frac{z_{-}^{2}}{2 \bar{\tau}}\right) Z(\tau, \bar{\tau}, z), \\
& \left.Z\right|_{(1 / 2,-3 / 2)} T=\varepsilon(T) Z(\tau, \bar{\tau}, z) .
\end{aligned}
$$


Using (4.4) and that $\frac{1}{6} p^{3}+\frac{1}{12} c_{2} \cdot p \in \mathbb{Z}$ by the index formula, we deduce that $\varepsilon(T)=e\left(-\frac{c_{2} \cdot p}{24}\right)$. Consistency of modular transformations requires that the $S$-transformation is also accompanied by a unitary pre-factor $\varepsilon(S)$. Since both $(S T)^{3}=-\mathbf{1}$ and $S^{2}=-\mathbf{1}$ leave $\tau$ invariant, $\varepsilon(S)=\varepsilon(T)^{-3}$. The phases for general $\gamma$ are denoted by $\varepsilon(\gamma)$.

As in Section 2, this SCFT also contains a spectral flow symmetry which implies a quasi-periodicity of the partition function as a function of $z^{a}$. This symmetry relates a state with charge $q$ to a state with charge $q+k$, where $k \in \Lambda$. Spectral flow determines in this way equivalence classes for the charges $q \in p / 2+\Lambda^{*}$. The collection of all coset representatives forms the discriminant group $\Lambda^{*} / \Lambda$, which is finite and abelian. Its order $\left|\Lambda^{*} / \Lambda\right|$ divides $D=\operatorname{det} d_{a b}$. The representatives $\mu$ are glue vectors and chosen such that they have minimal absolute length $\left|\mu^{2}\right|$. Spectral flow as a symmetry of the spectrum determines that the degeneracies $c\left(q_{\overline{0}}, q_{a}\right)$ depend only on the equivalence class of $q_{a}$ in $\Lambda^{*} / \Lambda+p / 2$ and $q_{\overline{0}}+\frac{1}{2} q^{2}$. The part of the spectrum with $q_{\overline{0}}+\frac{1}{2} q^{2}<0$ is the polar spectrum in this case. The spectral flow or quasi-periodicity combined with (4.5) allows us to perform a decomposition of $Z(\tau, \bar{\tau}, z)$ into a vector-valued modular form $h_{\mu}(\tau)$ and theta functions $\Theta_{\mu}(\tau, \bar{\tau}, z)$

$$
Z(\tau, \bar{\tau}, z)=\sum_{\mu \in \Lambda^{*} / \Lambda} \overline{h_{\mu}(\tau)} \Theta_{\mu}(\tau, \bar{\tau}, z)
$$

Note that this decomposition claims that $h_{\mu}(\tau)$ is a (weakly holomorphic) function of $\tau$. All the dependence on $\tau$ and $z$ of $Z(\tau, \bar{\tau}, z)$ is captured by the $\Theta_{\mu}(\tau, \bar{\tau}, z)$. The theta function corresponds to the $U(1)^{b_{2}}$-sector of the theory, which are the so-called singleton degrees of freedom [43-45] from the point of view of $\mathrm{AdS}_{3}$-supergravity. The expansions of $h_{\mu}(\tau)$ and $\Theta_{\mu}(\tau, \bar{\tau}, z)$ are

$$
h_{\mu}(\tau)=\sum_{n=0}^{\infty} c_{\mu}(n) q^{n-\Delta_{\mu}},
$$

$\Theta_{\mu}(\tau, \bar{\tau}, z)=\sum_{k \in \Lambda+p / 2}(-1)^{p \cdot(k+\mu)} e\left(\tau(k+\mu)_{+}^{2} / 2+\bar{\tau}(k+\mu)_{-}^{2} / 2+(k+\mu) \cdot z\right)$,

where $\Delta_{\mu} \in \mathbb{Q}$ and generically positive. It is given by

$$
\Delta_{\mu}=\frac{c_{R}}{24}-\left(\frac{\mu^{2}+p \cdot \mu}{2}-\left\lfloor\frac{\mu^{2}+p \cdot \mu}{2}\right\rfloor\right)
$$


such that $n-\Delta_{\mu}$ takes values in $\frac{c_{2} \cdot p}{24}+\frac{1}{2}(\mu+p / 2)^{2} \bmod \mathbb{Z}$.

The theta functions transform among each other under modular transformations. If we define

$$
\begin{aligned}
\left.\Theta_{\mu}\right|_{\left(b_{2}^{+}, b_{2}^{-}\right)} \gamma= & j(\gamma, \tau)^{-b_{2}^{+} / 2} j(\gamma, \bar{\tau})^{-b_{2}^{-} / 2} e\left(-\frac{c z_{+}^{2}}{c \tau+d}-\frac{c z_{-}^{2}}{c \bar{\tau}+d}\right) \\
& \times \Theta_{\mu}\left(\gamma(\tau), \gamma(\bar{\tau}), \frac{z_{+}}{c \tau+d}+\frac{z_{-}}{c \bar{\tau}+d}\right)
\end{aligned}
$$

then the $S$ - and $T$-transformation are given by

$$
\begin{aligned}
\left.\Theta_{\mu}\right|_{\left(b_{2}^{+}, b_{2}^{-}\right)} S & =\frac{1}{\sqrt{\left|\Lambda^{*} / \Lambda\right|}}(-i)^{\left(b_{2}^{+}-b_{2}^{-}\right) / 2} e\left(-\frac{p^{2}}{4}\right) \sum_{\delta \in \Lambda^{*} / \Lambda} e(-\delta \cdot \mu) \Theta_{\delta}(\tau, \bar{\tau}, z), \\
\left.\Theta_{\mu}\right|_{\left(b_{2}^{+}, b_{2}^{-}\right)} T & =e\left(\frac{(\mu+p / 2)^{2}}{2}\right) \Theta_{\mu}(\tau, \bar{\tau}, z),
\end{aligned}
$$

with $b_{2}^{+}=1$ and $b_{2}^{-}=b_{2}-1$, because the signature of the lattice is $\left(1, b_{2}-\right.$ $1)$. Note that $p^{2}$ is actually $p^{3}$, since the inner product is given by $d_{a b c} p^{c}$. However, since we are working here with quadratic forms, we use the notation $p^{2}$. The transformations of $\Theta_{\mu}$ for generic elements $\gamma \in \Gamma$ can be derived using the special properties of these theta functions, following for example $[46] .{ }^{6}$ Since $\mu^{2} \in \mathbb{Q}$ for every $\mu \in \Lambda^{*} / \Lambda$, an integer $m$ exists such that $\left.\Theta_{\mu}\right|_{\left(b_{2}^{+}, b_{2}^{-}\right)} T^{4 m}=\Theta_{\mu}(\tau, \bar{\tau}, z)$ for every $\mu \in \Lambda^{*} / \Lambda$. If $\gamma=\left[\begin{array}{ll}a & b \\ c & d\end{array}\right]$ with $a, d$ odd, and $c=0 \bmod 4 m$, then

$$
\left.\Theta_{\mu}\right|_{\left(b_{2}^{+}, b_{2}^{-}\right)} \gamma=\varphi(\mu, \gamma) \Theta_{a \mu}(\tau, \bar{\tau}, z)
$$

with

$$
\varphi(\mu, \gamma)=d^{-b_{2} / 2} e\left(\frac{d-1}{4} p^{2}+\frac{a b}{2}\left(\mu^{2}+\mu \cdot p\right)\right) \sum_{\ell \in \Lambda / d \Lambda} e\left(\frac{b}{2 d}(p / 2+\ell)^{2}\right)
$$

The sum over $\ell \in \Lambda / d \Lambda$ can be evaluated using Gauss sums, from which one can derive that $\left.\Theta_{\mu}\right|_{\left(b_{2}^{+}, b_{2}^{-}\right)} \gamma=\Theta_{\mu}(\tau, \bar{\tau}, z)$ with $\gamma \in \Gamma(4 m)^{*}$ for some $m$.

The weight of the theta functions $\frac{1}{2}\left(1, b_{2}-1\right)$ works out nicely with the weakly holomorphicity of $h_{\mu}(\tau)$. They have weight $\left(-\frac{b_{2}}{2}-1,0\right)$ and

${ }^{6} \mathrm{I}$ am grateful to the referee for pointing out this reference to me. 
transform under $S$ and $T$ as

$$
\begin{aligned}
& \left.h_{\mu}\right|_{-1-b_{2} / 2} S=-\frac{1}{\sqrt{\left|\Lambda^{*} / \Lambda\right|}}(-i)^{-b_{2} / 2-1} \varepsilon(S)^{*} e\left(-\frac{p^{2}}{4}\right) \sum_{\delta \in \Lambda^{*} / \Lambda} e(-\delta \cdot \mu) h_{\delta}(\tau), \\
& \left.h_{\mu}\right|_{-1-b_{2} / 2} T=\varepsilon(T)^{*} e\left(\frac{(\mu+p / 2)^{2}}{2}\right) h_{\mu}(\tau) .
\end{aligned}
$$

The additional --sign, appearing in the $S$-transformation, is a consequence of the unitary factor in (4.14). The vector $h_{\mu}(\tau)$ has length $\left|\Lambda^{*} / \Lambda\right|$. Similarly to the discussion around (2.18), some elements of the vector are required to be identical. This is again deduced from the transformation of $Z(\tau, \bar{\tau}, z)$ under $S^{2}=-\mathbf{1}$

$$
Z(\tau, \bar{\tau},-z)=\varepsilon(S)^{2} Z(\tau, \bar{\tau}, z)
$$

Moreover, $-\mathbf{1}$ acts on $\Theta_{\mu}(\tau, \bar{\tau}, z)$ by

$$
\Theta_{\mu}(\tau, \bar{\tau},-z)=(-1)^{p^{3}} \Theta_{\nu}(\tau, \bar{\tau}, z), \quad \nu=-\mu \quad \bmod \Lambda
$$

Since $\mathbf{- 1}$ acts trivially on $h_{\mu}(\tau)$, these equations determine that

$$
h_{\mu}(\tau)=h_{\nu}(\tau), \quad \nu=-\mu \quad \bmod \Lambda
$$

Thus the dimension $d$ of the vector $h_{\mu}(\tau)$ equals the number of orbits in $\Lambda^{*}$ by the group $\Lambda \otimes \mathbb{Z}_{2}$. Here $\Lambda$ acts additively and $\mathbb{Z}_{2}$ is multiplication by $\pm \mathbf{1}$. These orbits do not naturally form a group. For a one-dimensional lattice with inner-product $\ell, d=\frac{1}{2} \ell+1$ for $\ell$ even and $d=\frac{1}{2}(\ell+1)$ if $\ell$ is odd.

Since the individual $\Theta_{\mu}(\tau, \bar{\tau}, z)$ are forms of a congruence subgroup, and $\varepsilon(\gamma)$ is just the multiplier system of a power of $\eta(\tau)$, the functions $h_{\mu}(\tau)$ are (weakly holomorphic) modular forms of a congruence subgroup $\Gamma(4 m)$ for some $m .^{7}$ This shows that the formulas (3.9) and (3.10), which we used to calculate the number of constraints in Section 3, are also applicable here. The $\Delta_{\mu}$ in the expansion (4.10) play the role of $\lambda_{j}$ in (3.10). The form of $\Delta_{\mu}$ in (4.12) indicates that $h_{\mu}(\tau)$ is naturally written as $f_{\mu}(\tau) / \eta(\tau)^{c_{R}}$. The dimension of the space of weakly holomorphic $h_{\mu}(\tau)$ can then be calculated as the space of holomorphic $f_{\mu}(\tau)$ by $(3.9)$, if $c_{R} \geq b_{2}+2$. These $f_{\mu}(\tau)$

\footnotetext{
${ }^{7}$ Note that the level arising for the $\Theta_{\mu}(\tau, \bar{\tau}, z)$ might differ from the level of this congruence subgroup, since the transformation properties are slightly different.
} 
might capture interesting data as in the case of $\mathrm{SU}(2) \mathcal{N}=4$ Yang-Mills theory on $\mathbb{C P}^{2}$, see the last part of this section for more details.

It is interesting to compare the ratio between the number of polar coefficients and constraints. The number of polar terms $p\left(\mathbf{M}, c_{R}\right)$ is given by

$$
p\left(\mathbf{M}, c_{R}\right)=\sum_{\mu \in \Lambda^{*} / \Lambda \otimes \mathbb{Z}_{2}}\left\lceil\Delta_{\mu}\right\rceil=-\frac{1}{2} S(\mathbf{M})+\sum_{\mu \in \Lambda^{*} / \Lambda \otimes \mathbb{Z}_{2}}\left\{\Delta_{\mu}-\left(\left(\Delta_{\mu}\right)\right)+\frac{1}{2}\right\},
$$

where $S(\mathbf{M})$ is the number of terms $\Delta_{\mu}$ which take their value in $\mathbb{Z}$. Note that $p\left(\mathbf{M}, c_{R}\right)$ grows as $\frac{c_{R}}{24} d$ for large $c_{R}$, whereas the obstruction space grows as $\frac{4+b_{2}}{24} d$. Therefore, as in the case of $\mathcal{N}=(2,2)$ elliptic genera, the ratio between the number of polar terms and the obstructions is proportional to the central charge. Another resemblance is the appearance of the quantity $A_{\mathrm{p}}$ in the number of polar terms. The contribution of the triple intersection number to $c_{R}$ leads to many polar coefficients, therefore, the constraints are not very restrictive. A general analysis can be carried out, but instead only some comments on specific examples are given here. For one M5-brane on the hyperplane section of the quintic Calabi-Yau (considered in [17,37]), one finds seven polar terms and no constraints; for two M5-branes, the number of polar terms has already increased to 36 and then also one constraint is present. Gaiotto and Yin [37] analyzes a number of other situations where an M5-brane wraps a surface in a Calabi-Yau with $b_{2}=1$. Interestingly, for an M5-brane wrapping the hyperplane section of the bicubic in $\mathbb{C P}^{5}$ is reported that six basis elements suffice to determine the elliptic genus, whereas the number of polar coefficients is seven. Indeed, one can show with the above described technique that one constraint is present for this example. In agreement with [37], no constraints are found in the other examples worked out there.

The constraints are stronger in the case of twisted $\mathcal{N}=4$ supersymmetric Yang-Mills theory on a 4-manifold $M$. The coupling constant $g$ and the $\theta$-angle of the theory are conveniently combined in the complex coupling constant $\tau=\frac{\theta}{2 \pi}+\frac{4 \pi i}{g^{2}}$. This theory is invariant under a strong-weak coupling duality ( $S$-duality) [41], except that the gauge group $G$ of the theory is exchanged with the dual magnetic group $\hat{G}$ [42]. For example, the dual group of $\mathrm{SU}(\mathrm{N})$ is $\mathrm{SU}(N) / \mathbb{Z}_{N}$. When certain conditions are satisfied, the partition function is the generating function for the Euler numbers of instanton moduli spaces [36]. The $S$-duality manifests itself as modular behavior of the partition function of the twisted theory. Vafa and Witten [36] explain that the partition functions for gauge group $\mathrm{SU}(N) / \mathbb{Z}_{N}$, with different 't 
Hooft fluxes valued in $\mathbb{Z} / \mathbb{Z}_{N}$, transform among each other as a vector-valued modular form with weight $-\chi(M) / 2$. The partition function of $\mathrm{SU}(N)$ is given by the one of $\mathrm{SU}(N) / \mathbb{Z}_{N}$ with trivial 't Hooft flux, multiplied by $N^{-1+b^{1}}$ (with $b^{1}$ the first Betti number).

Remarkably, the transformation properties (4.17) closely resemble the transformation properties of the $\mathrm{SU}(N) / \mathbb{Z}_{N}$ partition functions of twisted $\mathcal{N}=4$ Yang-Mills theory. Both the $S$ - and $T$-transformation are compatible. This resemblance shows that the $\mathrm{SU}(N) / \mathbb{Z}_{N}$ partition functions can be combined into a single partition function by adding $U(1)$ degrees of freedom. This gives the partition function of the theory with gauge group $U(N)$, whose magnetic group is $U(N)$ as well. The space of constraints is more restrictive in this situation than for $\mathcal{N}=(4,0)$ SCFTs, since the number of polar degeneracies is much smaller now the contribution to the central charge of the triple intersection number vanishes. In the following, the number of polar terms and obstructions are calculated for the case of $\mathbb{C P}^{2}$, which gives the dimension of the space of weakly holomorphic modular forms with the required transformation properties. A determination of the polar coefficients, which might involve the so-called gap condition [36], is not attempted.

Since $b_{2}=1$ for $\mathbb{C P}^{2}$, the lattice $\Lambda$ is one-dimensional and the theta function is holomorphic in $\tau$. Consequently, $h_{\mu}(\tau)$ has weight $-\frac{3}{2}$. The second Chern class of $\mathbb{C P}^{2}$ is $3 J^{2}$, with $J$ the hyperplane class. The central charge $c_{R}$ from the SCFT reduces to the combination $\chi\left(\mathbb{C P}^{2}\right) N=3 N$. The unitary factor $\varepsilon(T)$ is then given by $\varepsilon(T)=e\left(\frac{N}{8}+\frac{c_{R}}{24}\right)=e\left(\frac{N}{4}\right)$. Note that the index formula cannot be used in this situation and that, therefore, $\varepsilon(T) \neq e\left(-\frac{c_{2} \cdot N}{24}\right)$. The theta functions $\Theta_{N, \mu}$ are given by

$$
\Theta_{N, \mu}(\tau, z)=\sum_{k \in \mathbb{Z}} e\left(\frac{\tau}{2 N}\left(\frac{N}{2}+\mu+k N\right)^{2}+\left(\frac{N}{2}+\mu+k N\right)\left(z+\frac{1}{2}\right)\right) .
$$

From the transformation properties of $\Theta_{N, \mu}$ follows that $h_{\mu}(\tau)$ transforms as

$$
\begin{aligned}
& \left.h_{\mu}\right|_{-1-b_{2} / 2} S=-\frac{1}{\sqrt{N}}(-i \tau)^{-3 / 2} e\left(\frac{N}{2}\right) \sum_{\nu} e\left(-\frac{\mu \nu}{N}\right) h_{\nu}(\tau), \\
& \left.h_{\mu}\right|_{-1-b_{2} / 2} T=e\left(-\frac{N}{4}+\frac{1}{2 N}\left(\mu+\frac{N}{2}\right)^{2}\right) h_{\mu}(\tau) .
\end{aligned}
$$


The functions satisfy moreover $\Theta_{N, \mu}(\tau,-z)=(-)^{N} \Theta_{N,-\mu}(\tau, z)$ and $h_{\mu}(\tau)=$ $h_{-\mu}(\tau)$, such that $h_{\mu}(\tau)$ can be reduced to a vector of length $\frac{N}{2}+1$ if $N$ is even and $\frac{N+1}{2}$ if $N$ is odd. The elements $h_{\mu}(\tau)$ are forms of $\Gamma(2 N)$ for $N$ even and otherwise $\Gamma(8 N)$. The number of polar terms $p(N)$ is given by

$$
p(N)=\sum_{\mu}\left\lceil\frac{N}{8}-\left(\frac{\mu^{2}}{2 N}+\frac{\mu}{2}-\left\lfloor\frac{\mu^{2}}{2 N}+\frac{\mu}{2}\right\rfloor\right)\right\rceil .
$$

One can straightforwardly determine the properties of the obstruction forms $g_{\mu}(\tau)$. They have weight $3 \frac{1}{2}$, we denote their representation again by $\mathbf{M}$. A closed expression can be given for the dimension of the space of forms which satisfy the required properties for $h_{\mu}(\tau)$. Table 2 presents the number of polar coefficients $p(N)$ and constraints on the polar spectrum for $N=$ $1, \ldots, 10$. For large $N, p(N)$ grows as $\frac{1}{16} N^{2}$ and the number of constraints as $\frac{5}{48} N$.

A first observation is that $\operatorname{dim} S_{3 \frac{1}{2}, \mathbf{M}}$ does grow more irregularly than the dimension of the space of cusp forms presented in Table 1. Table 2 confirms earlier results for $N=1$ and $N=2$, which are derived using the Weil conjectures $[47,48]$. For $N=1$, the space of potential partition functions is one-dimensional, therefore, $h_{0}(\tau)$ must be proportional to $\eta(\tau)^{-3}$. This agrees with the computation in [47]. For $N=2$, the table teaches us that no weakly holomorphic function $h_{\mu}(\tau)$ exists with the required properties. The obstruction form is given explicitly in [11]. The fact that no modular weakly holomorphic partition function exists is consistent with the known partition function calculated in [48]. This non-modular partition function has the form $f_{\mu}(\tau) / \eta(\tau)^{6}[36]$, where $f_{\mu}(\tau)$ are regularized Eisenstein series of weight $\frac{3}{2}$. The Fourier coefficients $c_{\mu}(n)$ of $f_{\mu}(\tau)$ are the number of equivalence classes of all positive definite forms, with discriminant $-4 n$ and $-4 n+1$ for $\mu=0$ and $\mu=1$, respectively. A connection between class numbers and rank 2 bundles over $\mathbb{C P}^{2}$ was earlier established by Klyachko [49]. The holomorphic $f_{\mu}(\tau)$ are not modular covariant, but can be made so by a non-holomorphic addition [50,51], similar to the way $E_{2}(\tau)$ can be made modular covariant.

Table 2: For $U(N)$ gauge theory on $\mathbb{C P}^{2}$, the number of polar coefficients $p(N)$ and constraints on the polar spectrum $\operatorname{dim} S_{3 \frac{1}{2}, \mathbf{M}}$ are listed for $N=1 \ldots 10$.

\begin{tabular}{llllllllllr}
\hline$N$ & 1 & 2 & 3 & 4 & 5 & 6 & 7 & 8 & 9 & 10 \\
$p(N)$ & 1 & 1 & 1 & 1 & 3 & 4 & 4 & 5 & 7 & 8 \\
$\operatorname{dim} S_{3 \frac{1}{2}, \mathbf{M}}$ & 0 & 1 & 0 & 0 & 1 & 1 & 0 & 0 & 1 & 2 \\
\hline
\end{tabular}


It is unclear how and if this holomorphic anomaly extends to larger values of $N$. Minahan et al. [52] explain how it does if the 4-manifold is $\frac{1}{2} \mathrm{~K} 3$.

The table shows that appropriate weakly holomorphic forms exists and are unique up to an overall factor for $N=3$ and $N=4$. One can show that for $N=3$, the $h_{\mu}(\tau)$ are given by

$$
h_{\mu}(\tau)=\frac{1}{2} \frac{\theta_{2}^{5}(\tau) \Theta_{3, \mu}\left[\begin{array}{l}
\frac{1}{2} \\
0
\end{array}\right](\tau)+\theta_{3}^{5}(\tau) \Theta_{3, \mu}\left[\begin{array}{l}
0 \\
0
\end{array}\right](\tau)+\theta_{4}^{5}(\tau) \Theta_{3, \mu}\left[\begin{array}{l}
0 \\
\frac{1}{2}
\end{array}\right](\tau)}{\eta(\tau)^{9}},
$$

where $\Theta_{N, \mu}\left[\begin{array}{l}a \\ b\end{array}\right](\tau)$ is defined by

$$
\Theta_{N, \mu}\left[\begin{array}{l}
a \\
b
\end{array}\right](\tau)=\sum_{k \in \mathbb{Z}} e\left(\frac{\tau}{2 N}(a N+\mu+k N)^{2}+(a N+\mu+k N) b\right) .
$$

The overall factor of $\frac{1}{2}$ in (4.25) is such that the first coefficient of the expansion is 1 . For $N=4, h_{\mu}(\tau)$ can be written in the form $f_{\mu}(\tau) / \eta(\tau)^{12}$, where $f_{\mu}(\tau)$ is a holomorphic vector-valued modular form.

It would be interesting to learn whether these functions give the Euler numbers of the instanton moduli spaces on $\mathbb{C P}^{2}$. This could shed light on the question of the holomorphic anomaly as well as the gap condition. It would also be instructive to find out whether the Euler numbers for $N=3$ and $N=4$ capture any special information, as is the case for $N=2$ where they are related to class numbers.

\section{Conclusion}

The dimension of the space of $\mathcal{N}=(2,2)$ and $(4,0)$ elliptic genera is shown to be equal to the number of independent degeneracies of the polar spectra, minus a number of constraints. This excludes certain polar spectra of SCFTs, since not all spectra are consistent with modular transformations. The constraints are also of interest from the point of view of $\mathrm{AdS}_{3}$ gravity, since the polar spectrum corresponds to the states which lie classically below the cosmic censorship bound. A technique is presented to calculate the number of constraints, which is generally applicable in situations where weakly holomorphic modular forms appear. As an additional example, $\mathcal{N}=4$ Yang-Mills on $\mathbb{C P}^{2}$ is discussed. This showed that weakly 
holomorphic modular forms do exist, which satisfy the modular properties expected for $\mathrm{SU}(3)$ and $\mathrm{SU}(4)$ partition functions. In a similar way, the technique might prove useful in future situations where one wants to determine specific modular forms.

From the mathematical point of view, the space of weak Jacobi forms of weight 0 and index $m\left(\tilde{J}_{0, m}\right)$ is studied. A Jacobi form can be decomposed in a vector-valued modular form $h_{\mu}(\tau)$ and theta functions $\theta_{m, \mu}(\tau, z)$ by quasi-periodicity. The dimension of $\tilde{J}_{0, m}$ is calculated as the number of polar coefficients of $h_{\mu}(\tau)$ minus the number of constraints imposed by the existence of a space of cusp forms. Also the generalization to $\operatorname{dim} \tilde{J}_{k, m}$ is given. In addition, the used techniques are applied to some generalizations of Jacobi forms, which arise in physics.

\section{Acknowledgments}

I am thankful to M.R. Gaberdiel, T. Gannon, G.B.M. van der Geer and G.W. Moore for comments and helpful discussions. I also would like to thank the workshop on "Three-Dimensional Quantum Gravity" organized by the McGill Center for High Energy Physics, the Department of Physics and Astronomy of Rutgers University and the Institute for Theoretical Physics of the ETH Zürich for their kind hospitality. My research is financially supported by the Foundation of Fundamental Research on Matter (FOM).

\section{References}

[1] J.L. Cardy, Operator content of two-dimensional conformally invariant theories, Nucl. Phys. B 270 (1986), 186.

[2] O. Aharony, S. Yankielowicz and A.N. Schellekens, Charge sum rules in $N=2$ theories, Nucl. Phys. B 418 (1994), 157, arXiv:hep-th/9311128.

[3] E. Witten, Elliptic genera and quantum field theory, Commun. Math. Phys. 109 (1987), 525.

[4] T. Eguchi, H. Ooguri, A. Taormina and S.K. Yang, Superconformal algebras and string compactification on manifolds with $S U(N)$ holonomy, Nucl. Phys. B 315 (1989), 193.

[5] V. Gritsenko, Elliptic genus of calabi-yau manifolds and jacobi and siegel modular forms, math/9906190. 
[6] A. Strominger and C. Vafa, Microscopic origin of the BekensteinHawking entropy, Phys. Lett. B 379 (1996), 99, arXiv:hep-th/9601029.

[7] J.M. Maldacena, A. Strominger and E. Witten, Black hole entropy in M-theory, JHEP 9712 (1997), 002, arXiv:hep-th/9711053.

[8] J.M. Maldacena, The large $N$ limit of superconformal field theories and supergravity, Adv. Theor. Math. Phys. 2 (1998), 231 (Int. J. Theor. Phys. 38 (1999) 1113), arXiv:hep-th/9711200.

[9] M. Cvetic and F. Larsen, Near horizon geometry of rotating black holes in five dimensions, Nucl. Phys. B 531 (1998), 239, arXiv:hepth/9805097.

[10] R. Dijkgraaf, J.M. Maldacena, G.W. Moore and E.P. Verlinde, A black hole farey tail, arXiv:hep-th/0005003.

[11] J. Manschot and G.W. Moore, A modern farey tail, arXiv:0712.0573 [hep-th].

[12] D. Niebur, Construction of automorphic forms and integrals, Trans. Amer. Math. Soc. 191 (1974), 373-385.

[13] M.R. Gaberdiel, S. Gukov, C.A. Keller, G.W. Moore and H. Ooguri, Extremal $\mathcal{N}=(2,2) 2 D$ conformal field theories and constraints of modularity, arXiv:0805.4216 [hep-th].

[14] M. Eichler and D. Zagier, The theory of Jacobi forms, Birkhäuser, 1985.

[15] N.P. Skoruppa, Über den Zusammenhang zwischen Jacobi-Formen und Modulformen halbganzen Gewichts, Dissertation, Universität Bonn, 1984.

[16] R. Minasian, G.W. Moore and D. Tsimpis, Calabi-Yau black holes and (0,4) sigma models, Commun. Math. Phys. 209 (2000), 325, arXiv:hepth/9904217.

[17] D. Gaiotto, A. Strominger and X. Yin, The M5-brane elliptic genus: modularity and BPS states, JHEP 0708, 070 (2007), arXiv:hepth/0607010.

[18] P. Kraus and F. Larsen, Partition functions and elliptic genera from supergravity, JHEP 0701 (2007), 002, arXiv:hep-th/0607138. 
[19] J. de Boer, M.C.N. Cheng, R. Dijkgraaf, J. Manschot and E. Verlinde, A farey tail for attractor black holes, JHEP 0611 (2006), 024 arXiv:hepth/0608059.

[20] T. Kawai, Y. Yamada and S.K. Yang, Elliptic genera and N=2 superconformal field theory, Nucl. Phys. B 414 (1994), 191, arXiv:hep-th/ 9306096.

[21] N. Koblitz, Introduction to elliptic curves and modular forms, SpringerVerlag, 1993.

[22] M.I. Knopp, Some new results on the Eichler cohomology of automorphic forms, Bull. Amer. Math. Soc. 80 (1974), 607-632.

[23] E. Witten, Three-dimensional gravity revisited, arXiv:0706.3359 [hep-th].

[24] P. Sarnak, Some applications of modular forms, Cambridge University Press, 1990.

[25] P. Griffiths and J. Harris, Principles of algebraic geometry, John Wiley and Sons, 1978.

[26] R.E. Borcherds, The Gross-Kohnen-Zagier theorem in higher dimensions, Duke Math. J. 97 (1999), 219-233.

[27] W. Eholzer and N.P. Skoruppa, Modular invariance and uniqueness of conformal characters, Commun. Math. Phys. 174 (1995), 117, arXiv:hep-th/9407074.

[28] P. Bantay and T. Gannon, Vector-valued modular functions for the modular group and the hypergeometric equation, arXiv:0705.2467 [math.NT].

[29] D. Zagier, The Eichler-Selberg trace formula on $S L_{2}(\mathbb{Z})$, Appendix in S. Lang Introduction to modular forms, Springer-Verlag, 1976; Correction in modular functions of one variable VI, Lect. in Math., 627, SpringerVerlag, 1976.

[30] T.M. Apostol, Introduction to analytic number theory. Springer-Verlag, 1976.

[31] N.P. Skoruppa and D. Zagier, Jacobi forms and a certain space of modular forms, Invent. Math. 94 (1988), 113. 
[32] N.P. Skoruppa and D. Zagier, A trace formula for Jacobi forms, J. Reine Angew. Math. 393 (1989), 168.

[33] N.P. Skoruppa, Memorandum on dimension formulas for spaces of Jacobi forms, arXiv:0711.0632 [math.NT].

[34] J. Kramer, A geometrical approach to the theory of Jacobi forms, Comp. Math. 79 (1991), 1.

[35] M.R. Gaberdiel and C.A. Keller, Modular differential equations and null vectors, arXiv:0804.0489 [hep-th].

[36] C. Vafa and E. Witten, A strong coupling test of $S$ duality, Nucl. Phys. B 431 (1994), 3, arXiv:hep-th/9408074.

[37] D. Gaiotto and X. Yin, Examples of M5-brane elliptic genera, JHEP 0711 (2007), 004, arXiv:hep-th/0702012.

[38] F. Denef and G.W. Moore, Split states, entropy enigmas, holes and halos, arXiv:hep-th/0702146.

[39] J.M. Maldacena, G.W. Moore and A. Strominger, Counting BPS black holes in toroidal type II string theory, arXiv:hep-th/9903163.

[40] D.S. Freed and E. Witten, Anomalies in string theory with D-branes, arXiv:hep-th/9907189.

[41] C. Montonen and D.I. Olive, Magnetic monopoles as gauge particles? Phys. Lett. B 72 (1977), 117.

[42] P. Goddard, J. Nuyts and D.I. Olive, Gauge theories and magnetic charge, Nucl. Phys. B 125 (1977), 1.

[43] E. Witten, AdS/CFT correspondence and topological field theory, JHEP 9812 (1998), 012, arXiv:hep-th/9812012.

[44] J.M. Maldacena, G.W. Moore and N. Seiberg, D-brane charges in fivebrane backgrounds, JHEP 0110 (2001), 005, arXiv:hep-th/0108152.

[45] G.W. Moore, Anomalies, Gauss laws, and page charges in M-theory, C. R. Phys. 6 (2005), 251, arXiv:hep-th/0409158.

[46] A. Ogg, Modular forms and Dirichlet series, W. A. Benjamin, Inc., 1969.

[47] L. Göttsche, The Betti numbers of the Hilbert scheme of points on a smooth projective surface, Math. Ann. 286 (1990), 193. 
[48] K. Yoshioka, The Betti numbers of the moduli space of stable sheaves of rank 2 on $\mathbb{P}^{2}$, J. Reine. Angew. Math. 453 (1994), 193.

[49] A.A. Klyachko, Moduli of vector bundles and numbers of classes, Funct. Anal. Appl. 25 (1991), 67.

[50] D. Zagier, Nombres de classes et formes modulaires de poids 3/2, C.R. Acad. Sci. Paris 281 (1975), 883.

[51] F. Hirzebruch and D. Zagier, Intersection numbers of curves on Hilbert modular surfaces modular forms of Nebentypus, Invent. Math. 36 (1976), 57.

[52] J.A. Minahan, D. Nemeschansky, C. Vafa and N.P. Warner, E-strings and $N=4$ topological Yang-Mills theories, Nucl. Phys. B 527 (1998), 581, arXiv:hep-th/9802168.

Institute FOR TheOreticAl Physics

UNIVERSITY OF AMSTERDAM

VALCKENIERSTRAAT 65

1018 XE, AMSTERdAM

The Netherlands

Current address:

New High Energy Theory Center

Rutgers University

PISCATAWAY

NJ 08854-8019

USA

E-mail address: manschot@physics.rutgers.edu

ReCEIVED June 11, 2008 
